\title{
An Improved Hough Transform Algorithm Based on Reduced Particle Swarm Optimization and its Applications in the Train Wheel Image Detection
}

\author{
Zengqiang $\mathrm{Ma}^{1}$, Xiaoyun Liu ${ }^{1}$, Zheng Liu ${ }^{1}$, Sha Zhong ${ }^{1}$ and Yusi Zhang ${ }^{1,2}$ \\ ${ }^{1}$ School of Electrical and Electronics Engineering, Shijiazhuang Tiedao \\ University, Shijiazhuang, 050043, China \\ ${ }^{2}$ Department of Electrical and Information Engineering, Hebei Jiaotong \\ Vocational and Technical College, Shijiazhuang, 050035, China
}

mzqlunwen@126.com

\begin{abstract}
The Hough Transform algorithm based on RPSO (Reduced Particle Swarm Optimization) is widely used in image detection. But it has several defects, such as being apt to plunge a local extremum and low target detection precision. In order to overcome these defects of the original algorithm, an improvedalgorithm, which is updated with the mechanism of SA (simulated annealing), is presented in this paper. In the improved algorithm, the output parameters of the Hough Transform was regarded as particle positions, and the opposite value of accumulation avrorin Hough Transform was employed as a fitness function of RPSO algorthm. Because the mechanism of the SA was involved, the velocities and positions of the particles are updated in real-time in the process of the crossover and Gaussion mation Consequently, the ability of converging to global optimum solution is obvousty improved. Then, the comparison and analysis of the experiment results between the-original algorithm and the improved one have been carried out in the application of the train whee image detection. The experiment results demonstrate that the accuracy of image detection is evidently increased and the evolution speed is significantly boosted in the proposed algorithm, especially as the image has complex background or high level noise.
\end{abstract}

Keywords: Hough Transform, RPSO (Reduced Particle Swarm Optimization), SA (simulated annealing), crossoyer and Gaussian mutation

\section{Introduction}

The relative position between train wheel and rail can directly reflect contact state of the wheel-rail. Currently, image detection technology is used to achieve the on-line and continuous meásurement of train wheel-rail relative position, which is an important means to monitor the stability and safety of train, and research on it is of great significance for yehicle performance and derailment mechanism [1-3]. In image detection technology, Hough Transform, which is an important and popular method to recognize and detect defined curves [4-5], is proposed by Paul Hough in 1962. The essence of Hough Transform is the mapping of data points from image space into parameter space. Because of its robustness, Hough Transform has been applied widely in computer vision and pattern recognition area [6-7].

The conventional Hough Transform is of heavy computation, due to the limitation [8] that the Hough Transform treats all angles equally and has less-salient peaks.In order to overcome this limitation, Galambos [9] proposed an improved algorithm, which reduced the computation and accelerated the speed by using gradient information; Matas [10] presented the Progressive Probabilistic Hough Transform (PPHT) which minimizes the 
computation by exploiting the difference in the fraction of votes needed to detect the lines with different number of supporting points reliably; Duquenoy and Taleb-Ahmed [11] presented the Hough Transform as a linear operation, and an image decomposition method was utilized to increase the processing speed and widen the Hough Transform's total perception of the image; literature [12] proposed a rigorous randomized Hough Transform; Yingtao Zhang [13] proposed the Hough Transform algorithm based on RPSO [14], which improved the accuracy and efficiency of Hough Transform.

In order to overcome the defects of being apt to plunge a local extremum and low target detection accuracy in the Hough transform algorithm based on RPSO. In this paper, an improved algorithm, which is updated with the mechanism of SA (simulated annealing) [15-16], is proposed in this paper. In the improved algorithm, SA is used to update velocities and positions of particle swarm in real-time in the process of the crossover and Gaussian mutation. Combining the fast search optimum ability of RPSO with probability jump property of SA, the improved algorithm can converge to global optimum solution quickly. The experiment results show that the evolution speed is significantly boosted and detection accuracy is evidently increased after the original algorithm was substiated by the improved one.

\section{Principle and Defect of the Hough Transform Algorithm Based on RPSO}

\subsection{Principle of the Original Algorithm}

Hough transform algorithm based on RPSQ combines the RPSO algorithm and Hough transform. In the algorithm, the output parameters of the Hough Transform was regarded as particle positions, and the value oraccumatation array in Hough Transform was employed as a fitness function of RPSO algorithm. Actually, the RPSO algorithm is an improved particle swarm optimization algorithm to search the global optimal value. In the RPSO algorithm, the positions fitness vanues are calculated and sorted in a list with descending order. 'Stronger' particles whose fitness values are in the higher positions of the list are preserved, and the reduced particles are applied to search the best positions. It is assumed that the Hough transform algorithm based on RPSO is employed for line detection, its operation principle is shown as Figure1.

$$
\begin{gathered}
f\left(\rho_{k}, \theta_{k}\right)= \begin{cases}1 & \rho_{\mathrm{k}}=\rho_{0}, \theta_{\mathrm{k}}=\theta_{0} \\
0 & \rho_{\mathrm{k}} \neq \rho_{0}, \theta_{\mathrm{k}} \neq \theta_{0}\end{cases} \\
P_{i n}(k+1)= \begin{cases}P_{i n}(k) & f\left(P_{i n}(k)\right)>f\left(x_{i n}(k)\right) \\
x_{i n}(k) & f\left(P_{i n}(k)\right) \leq f\left(x_{i n}(k)\right)\end{cases} \\
v_{\mathrm{id}}(k+1)=w * v_{\mathrm{id}}(k)+c_{1} r_{1}\left(p_{\mathrm{id}}(k)-x_{\mathrm{id}}(k)\right)+c_{2} r_{2}\left(p_{\mathrm{gd}}(k)-x_{\mathrm{id}}(k)\right) \\
x_{\mathrm{id}}(k)=x_{\mathrm{id}}(k)+v_{\mathrm{id}}(k+1)
\end{gathered}
$$




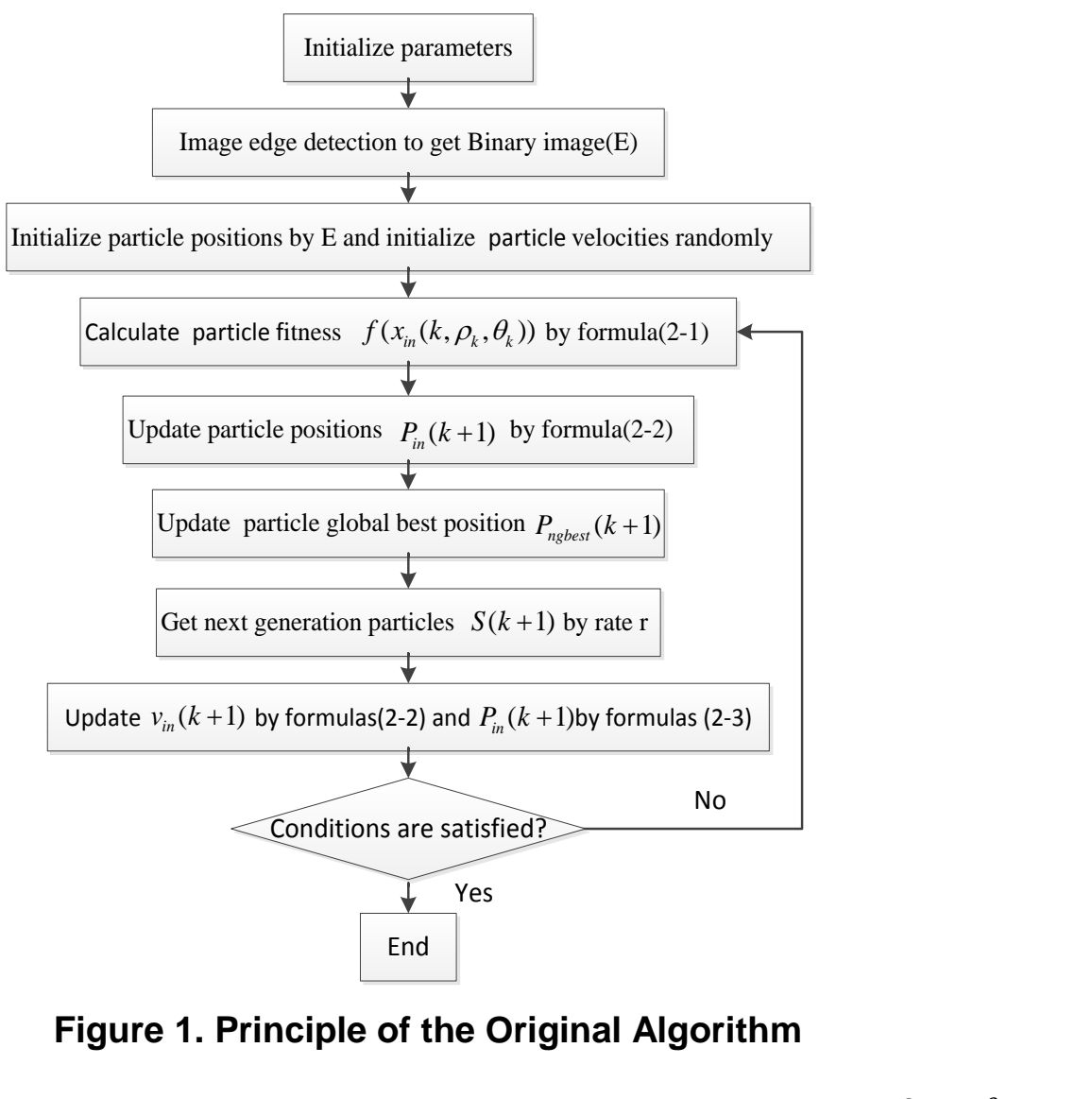

In the formulas, the output paameters of the Hough Transform include $\rho^{\rho}$ and $\theta$, and their initial values are $\rho_{0}$ and $\theta_{0}$ respectively $A$ is an two-dimensional accumulation array; ${ }^{f}$ is used to calculate the function fitness; ${ }^{x_{i n}}$ is the position of the particle in a ndimensional space at curnent time; $\mathcal{R}_{\text {in }}$ is the best position of the particles; ${ }^{w}$ is an inertia weight; $c_{1}$ and $c_{2}$ are the learning factors which are also called acceleration constant; ${ }^{r}$ and are the random number, and their value range are both[0,1]; ${ }^{k}$ is the number of iteration; $p_{i d}(k)$ and $p_{g d}(k)$ are personal best position of particles and group best position of particles respectively.

\subsection{Limitation of the Hough Transform Algorithm Based on RPSO}

The Hough Transform algorithm based on RPSO combines the Hough Transform with the RPSO algorithm. However, the RPSO algorithm falls into local minimum easily so that the original algorithm cannot get the global result quickly and accurately. Low accuacy for target detection is a fatal limitation of the original algorithm, the reason for which is that original algorithm fails to get the global result quickly and accurately, the following experiment is to illustrate the limitation of the original algorithm. In this experiment, the Hough Transform algorithm based on RPSO is utilized to detect train wheel image. Figure2 is the actual train wheel image; Figure 3 shows the detection results on different Gaussian noise $\sigma$ in the original algorithm; Figure 4 shows the detection results on different salt and pepper noise ${ }^{n}$ in the original algorithm. 


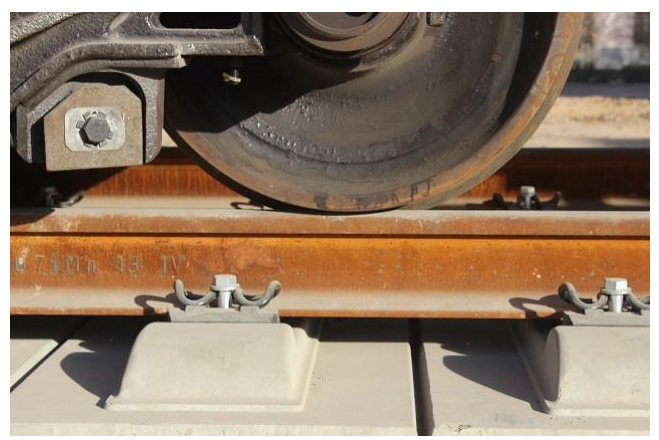

Figure 2. Actual Train Wheel Image

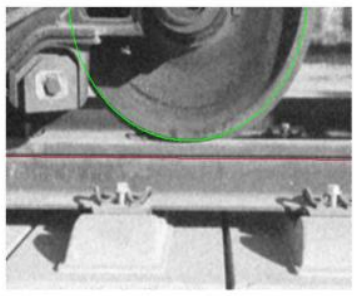

$\sigma=0.1$

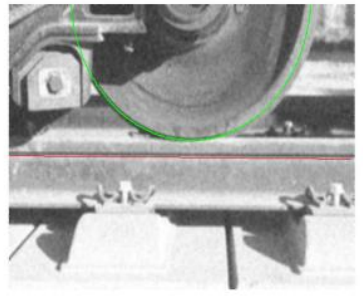

$\sigma=0.2$

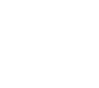

Figure 3. Detection Results on Different $\sigma$ in the Original Algorithm

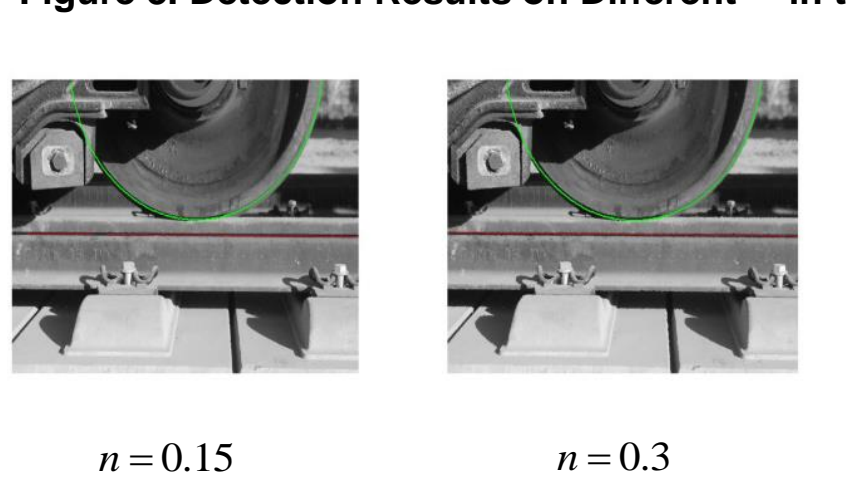

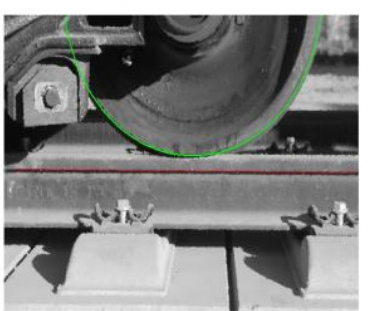

$n=0.45$

Figure 4 Detection Results on Different $n$ in the Original Algorithm

As it can be seen from the experiment, the detection results of the original algorithm are not very accurate, the reason for which is that the original algorithm fail to get the global result quickly and accurately. Therefore, improving the global search ability is a critical method to remedy the limitation of the original IMM algorithm.

\section{The Improved Hough Transform Algorithm Based on RPSO}

To overcome the limitation that the original Hough Transform algorithm based on RPSO fails to obtain the global result quickly and accurately, an improved Hough Transform algorithm based on RPSO, which combines the original algorithm with SA algorithm, is presented in this paper. In the improved algorithm, the output parameters of the Hough Transform was regarded as particle positions, and the opposite value of accumulation array in Hough Transform was employed as a fitness function of RPSO algorithm. Because the mechanism of the SA is involved, the velocities and positions of the particles are updated in real-time in the process of the crossover and Gaussian mutation. As a result, the ability of converging to global optimum solution is obviously improved. The following experiment results show that the limitation of the original 
algorithm has been remedied; the detection accuracy and evolution speed have been increased more obviously in the improved algorithm.

\subsection{Principle of the Improved Algorithm}

The improved Hough Transform algorithm based on RPSO combines the original algorithm and SA algorithm. With the temperature dropping from high to low, SA algorithm seeks the global excellent value of target function randomly on the basis of characteristic of jump probability. Not only is the optimal value accepted by the Metropolis but also the poor value. Consequently, velocities and positions of the particles are updated in real-time by SA algorithm, and the SA algorithm can jump out from the local extremism and find the global excellent value. The experiment results show that detection accuracy and evolution speed are evidently increased in the improved Hough Transform algorithm based on RPSO.

Supposing that the improved algorithm is employed for line detection, its operation principle is shown as Figure5. Compared with the original algorithm, crossover and mutation module and SA module are two newly added modules for updating the velocities and positions of the particles respectively in real-time, 'Stronger' particles whose fitness values are in the higher positions of the list are selected for searching the global excellent value. Based on those advantages of the two multiplied module mentioned above, the ability of searching the global excellent result is evidently increased in the improved algorithm.

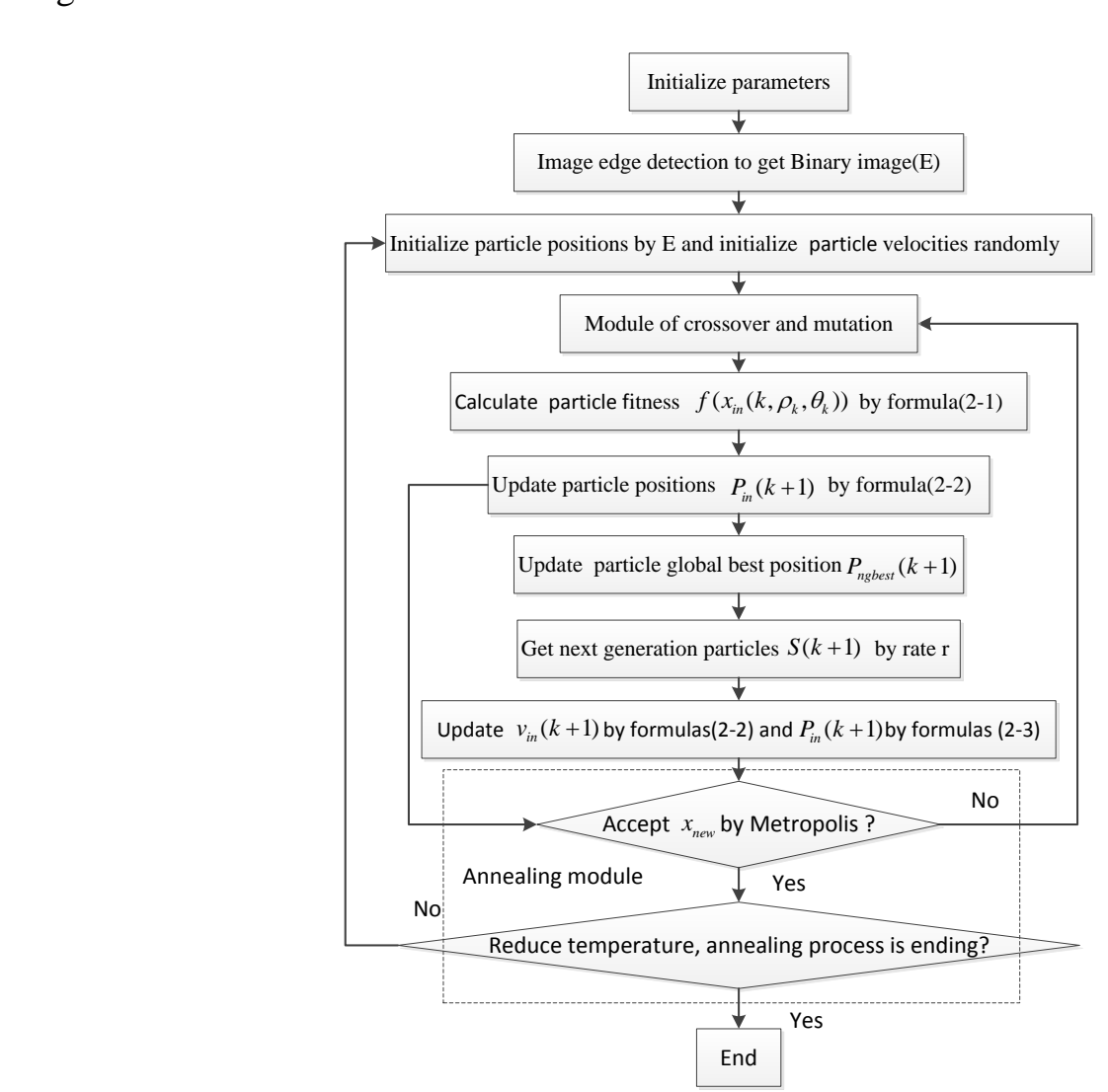

Figure 5. Principle of the Improved Algorithm 


\subsection{The Module of Crossover and Mutation}

The module of crossover and mutation include hybrid operation with SA algorithm and mutation operation with SA algorithm. In entire operation for module of crossover and mutation, the particle swarm is independently hybridized to get next sub-population and make them anneal firstly; then the new sub-population by Gaussian mutation operation is also simulated annealing to form the next optimal particle swarm.

\subsubsection{The Crossover Operation and Mutation Operation}

The crossover operation is that a fixed number of parent particles are crossed between each two single particles in closed chamber, but it must guarantee that the number of new produced child particles is the same as the parent particles. As the crossover operation occurs, the positions of the child particles are gained by the positions of the parent particles according to formulas(3-1),(3-2). The velocities of the child particles are also computed by the velocities of the parent particles according to formulas $(3-3),(34)$. In the formulas, $x$ is the D-dimensional position vector, $v$ is the D-dimensional velocity vector, ${ }^{p}$ is the D-dimensional vector of random distribution and its value range is $[0,1]$, arent $_{k}(x)$ and child $_{k}(x)$ are positions of the parent particles and child particles respectively, $\operatorname{parent}_{k}(v)$ and $\operatorname{child}_{k}(v)$ are velocities of the parent particles and child particles respectively.

$$
\begin{aligned}
& \operatorname{child}_{1}(x)=p^{*} \operatorname{parent}_{1}(x)+(1-p) * \text { parent }_{2}(x) \\
& \operatorname{child}_{2}(x)=p^{*} \text { parent }_{2}(x)+(1-p)^{*} \text { parent }_{1}(x) \\
& \operatorname{child}_{1}(v)=\frac{\text { parent }_{1}(v)+\text { parent }_{2}(v)}{\mid \operatorname{parent}_{1}(v)+\text { parent }_{2}(v) \mid} \mid \text { parent }_{1} \mid \\
& \operatorname{child}_{2}(v)=\frac{\operatorname{parent}_{1}(v)+\operatorname{parent}_{2}(v)}{\left|\operatorname{parent}_{1}(v)+\operatorname{parent}_{2}(v)\right|} \mid \text { parent }_{2} \mid
\end{aligned}
$$

In mutation operation, particles are firstly selected according to the mutation probability, and then these particies are evolved by gauss mutation operator and form a new generation of particle swarn. The gauss mutation is operated as follows:

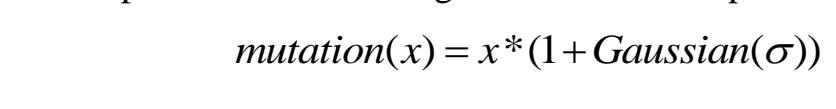

\subsubsection{Principle of the Improved Simulated Annealing Algorithm}

The principle of simulated annealing algorithm is as follows:

(1) Initialize the annealing temperature ${ }^{T_{0}}$, acceleration $a$ and generate an initial value $x_{0}$ se $x_{0}$ as the best value $x b e s t$, based on the parameters above, the target function $E\left(x_{x p e s t}\right)$ can be calculated as current target function value;

(2) Produce a new value ${ }^{x_{\text {new }}}$ near the current best value $x$ best and work out the new target function value $E\left(x_{\text {new }}\right)$, then calculate the changed value of target function $\Delta E$ between the $x_{\text {new }}$ and $x_{x b e s t}$ by formula $\Delta E=E\left(x_{\text {new }}\right)-E(x b e s t)$;

(3)Accept the new value ${ }^{x_{\text {new }}}$ according to the probability which is obtained through the formula $\min \left\{1, \exp \left(-\Delta E / T_{k}\right)\right\}>\varepsilon, \varepsilon$ is a random number and its value range is $[0,1]$;

(4)Go to step (5), if the sampling process is terminated on the basis of convergence standard; otherwise, return the step (2); 
(5)Reduce the temperature from $T$ to $a T$, and then go to step (2) to repeat the process until annealing process is ended, finally, output the optimal value.

In the simulated annealing algorithm, the annealing acceleration $a$ is a parameter and it has a great impact on the performance of the algorithm. There are two cases for $a$ affecting the algorithm, one of the cases is that when ${ }^{a}$ is very small, this algorithm tends to the optimal result with poor convergence velocity, the other is that when $a$ is too big, this algorithm cannot get the global excellent result and the detection accuracy is reduced. In order to reduce the impact of $a$ on the performance of the algorithm, the improved algorithm, which designs the function to change the temperature by formula (3-6), is presented in this paper. It can be seen from formula (3-6), the number of state accepted in the same temperature is a parameter to adjust the appropriate temperature, which enhances suitability of the algorithm for temperature. Compared with the original SA algorithm, the limitation of the original SA algorithm has been recuperated, the evolution velocity and detection accuracy have been increased more obviously in the improved algorithm.

$$
t_{k+1}=t_{k} e^{-c^{*} \alpha}, \alpha=\operatorname{acceptNum} /(\text { acceptNum }+ \text { totalNum })
$$

\subsubsection{Principle for the Module of Crossover and Mutation}

The module of crossover and mutation includes the crossover operation with SA algorithm and the mutation operation with SA algorithm. The principle for the module of crossover and mutation is as follows:

(1)The child particles are generated by the parent particles, which are crossed between each two single particles according to the cross probability $p_{c}$. The detailed operation is as follows:

Choose two particles $x_{i}, x_{i}$ randomly from the parent particles and make them crossed by formula(3-1),(3-2). The two child particles $x_{i}^{\prime},{ }^{\prime}{ }_{j}$ are emerged, then work out the target functions of $f\left(x_{\mathrm{i}}, f\left(x_{\mathrm{j}}\right), f\left(x_{i}^{\prime}\right), f\left(x_{j}^{\prime}\right)\right.$ and select two the optimal particles as new generation particles by formulas $(3-7),(3-8)$. In the formulas, ${ }^{\varepsilon}$ is random number and its value range is $[0,1]$.

(2)Based on the results step (1), the child particles are generated again by the parent particles, which are evolved according to the mutation-probability $P_{m}$. The detailed operation is as follow:

Based on the mutation-probability ${ }_{m}$, select single particle ${ }^{x_{i}}$ from the results of step(1) and make them Gaussian variation by formula(3-5), the new particle ${ }^{x_{i}}{ }_{i}$ is appeared, then calculate the target functions of $f\left(x_{\mathrm{i}}\right), f\left(x_{i}^{\prime}\right)$ and choose the optimal particle as new generation particle by formulas (3-7).

$$
\begin{aligned}
& \min \left\{1, \exp \left(-\left(f\left(x_{i}^{\prime}\right)-f\left(x_{i}\right)\right) / T\right)\right\}>\varepsilon \\
& \min \left\{1, \exp \left(-\left(f\left(x_{j}^{\prime}\right)-f\left(x_{j}\right)\right) / T\right)\right\}>\varepsilon
\end{aligned}
$$

\subsection{Principle for the Module of Simulated Annealing}

In order to search the global excellent result, the module of simulated annealing mainly utilizes the improved SA algorithm in Section 3.2.2 to optimize the particle swarm. In the improved SA algorithm, the fitness of two positions is to decide whether the new result is accepted or reduce the temperature. Meanwhile, due to adjust appropriate temperature by 
formula (3-6), the improved Hough Transform algorithm based on RPSO has higher evolution velocity and detect precision than the original algorithm.

\section{Experiment Results Comparison Between the Original Algorithm and the Improved One}

In order to testify performance of the proposed algorithm, in the following experiments, the actual train wheel image (Figure2) is used for image detection in the improved algorithm. Figure6 shows the detection results on different Gaussian noise $\sigma$ in the improved one; Figure7 shows the detection results on different salt and pepper noise ${ }^{n}$ in the improved one.

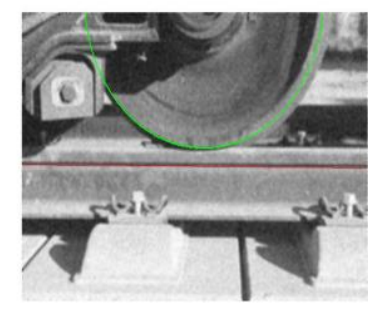

$\sigma=0.1$

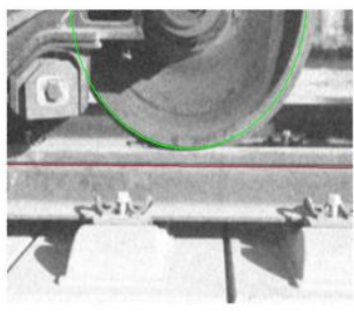

$\sigma=0.2$

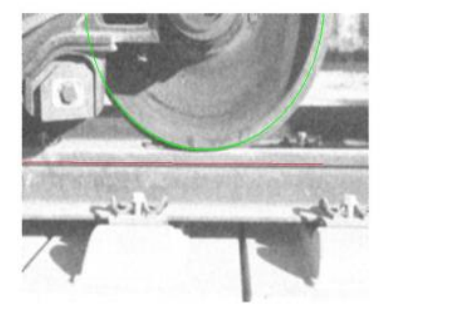

$\sigma=0.3$

Figure 6. Detection Results on Different $\sigma$ in the Original Algorithm

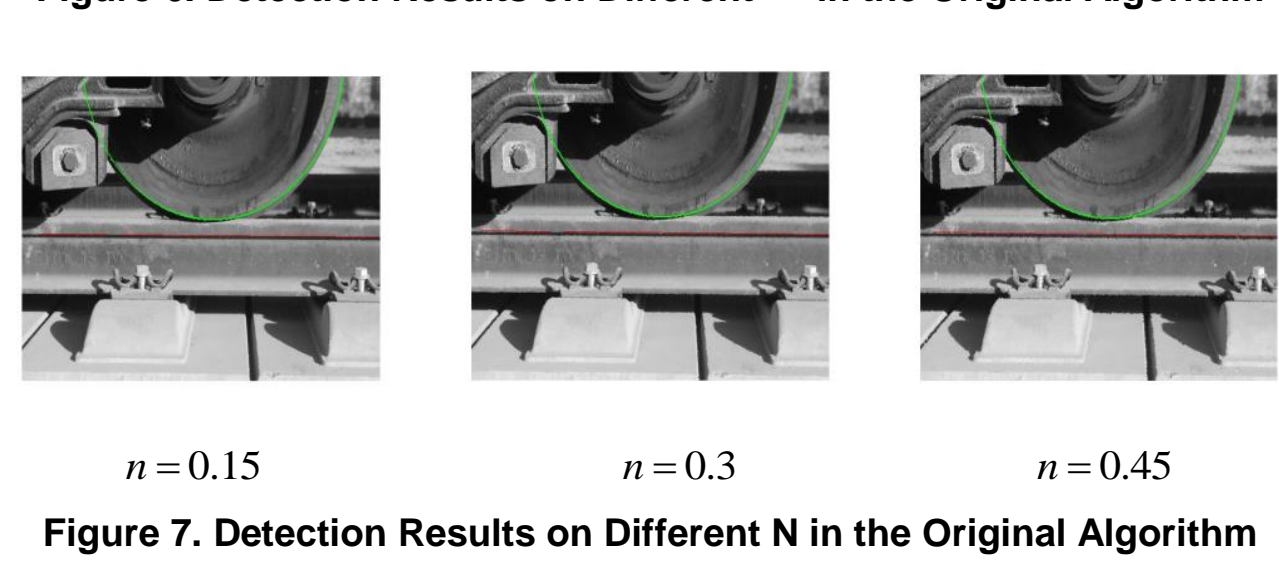

In order to evaluate the accuracy of the line detection results, a metric, error sum, is defined to measare the error and the difference between the detection result and true result. By the same way, the error sum for circle detection results is also defined. The error of line and circleare defined as follows:

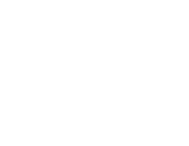

$$
\begin{gathered}
E_{\mathrm{l}}=\sum\left(\left|\rho_{\mathrm{d}}-\rho_{\mathrm{t}}\right|+\left|\theta_{\mathrm{d}}-\theta_{\mathrm{t}}\right|\right) \\
E_{\mathrm{c}}=\sum\left(\left|x_{\mathrm{d}}-x_{\mathrm{t}}\right|+\left|y_{\mathrm{d}}-y_{\mathrm{t}}\right|+\left|R_{\mathrm{d}}-R_{\mathrm{t}}\right|\right)
\end{gathered}
$$

Where, $\rho_{\mathrm{t}}$ and $\theta_{\mathrm{t}}$ are the true coordinates of line's polar radius and angle, while $\rho_{d}$ and $\theta_{d}$ are the detected coordinates of line's polar radius and angle respectively. Meanwhile, Where, $x_{\mathrm{t}}, y_{\mathrm{t}}$ and $R_{\mathrm{t}}$ are the true coordinates of circle's center and radius, while $x_{\mathrm{d}}, y_{\mathrm{d}}$ and $R_{\mathrm{d}}$ are the detected coordinates of circle's center and radius respectively. After the experiments are performed, the Chart 4-1 shows the detection time and error under the same Gaussian noise between the original algorithm and the improved one; Chart 4-2 shows the detection time and error under the same salt and pepper noise between the original algorithm and the improved one. 


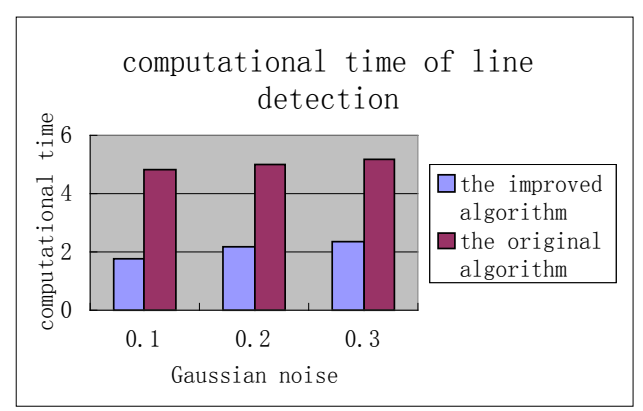

(a) Line Detection Time Comparison

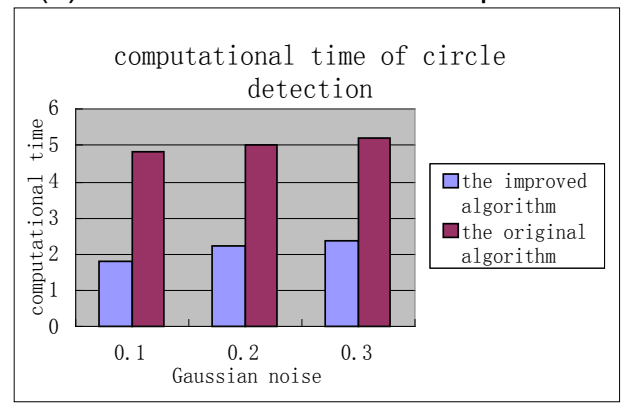

(c) Circle Detection Time Comparison

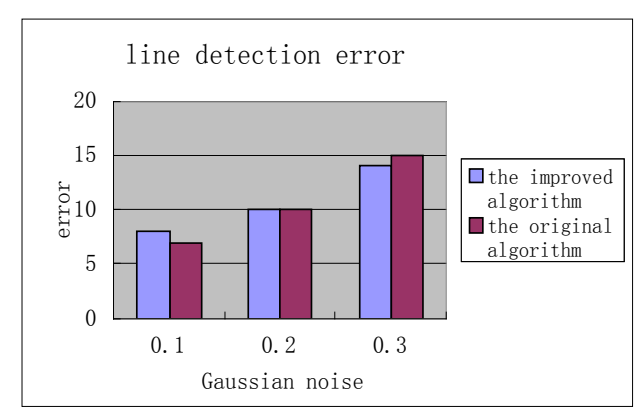

(b) Line Detection Error Comparison

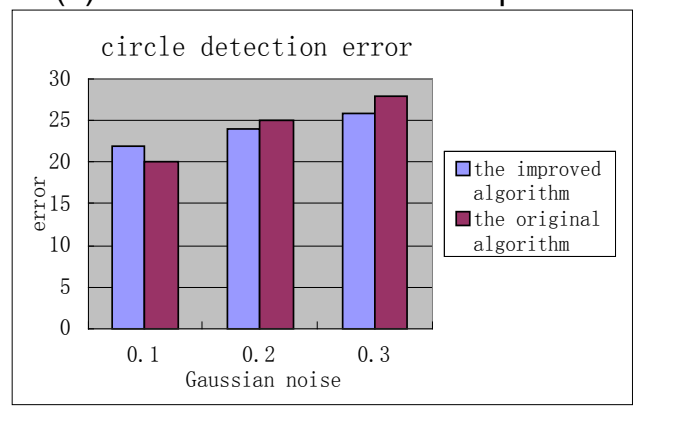

(d) Circle Detection Error Comparison

Chart 4-1. Comparison of Detection Results on Different $\sigma$ Between the Improved Algorithm and the Original One

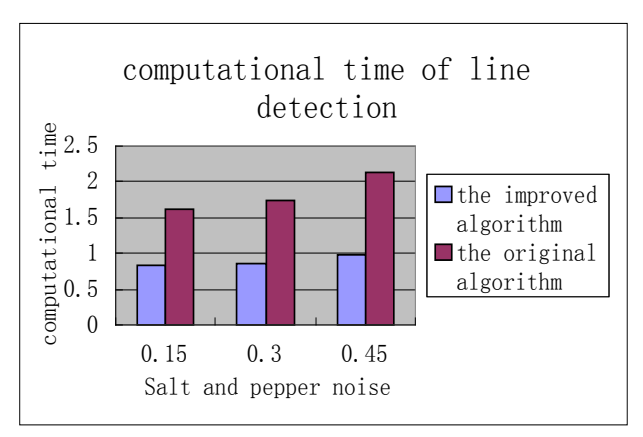

(a) Line Detection Jime Comparison

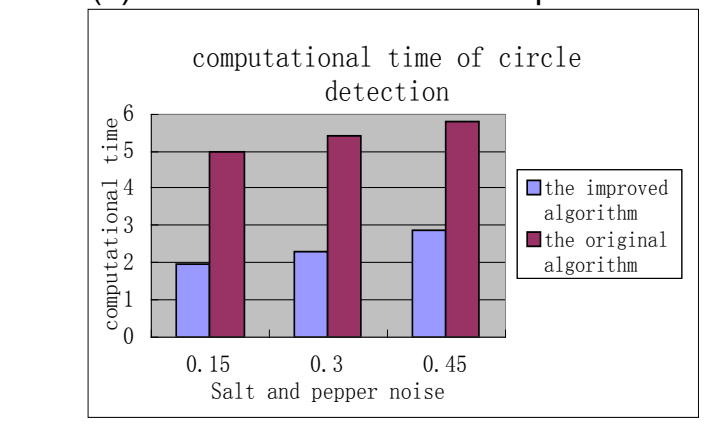

(c) Circle Detection Time Comparison

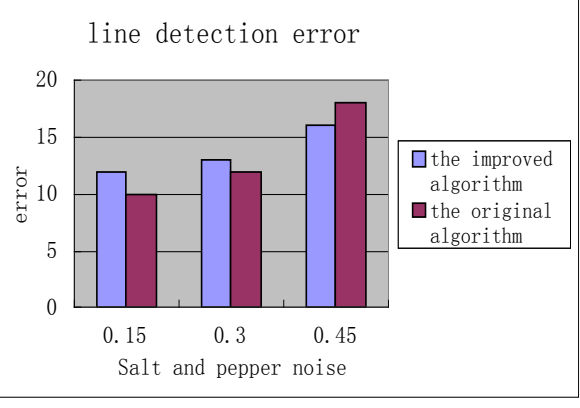

(b) Line Detection Error Comparison

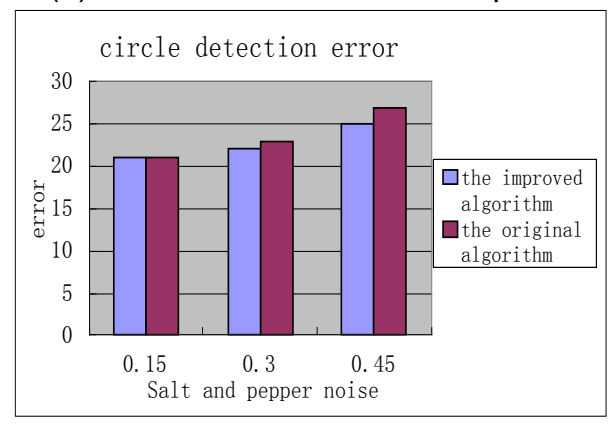

(d) Circle Detection Error Comparison

\section{Chart 4-2. Comparison of Detection Results on Different $n$ between the Improved Algorithm and the Original One}

From the experiment results, it can be seen that the improved hough transform algorithm based on RPSO detects the curves more precisely and spends less 
computational time, especially for images with complex background or with high level noise.

\section{Conclusions}

After the principle of the original Hough Transform algorithm based on RPSO for target detection is introduced and its defects are analyzed, an improved Hough Transform algorithm based on RPSO is proposed in this paper. In the improved algorithm, the SA algorithm is involved, the velocities and positions of particles are updated in real-time through crossover and mutation module and simulated annealing module respectively. Consequently, the global optimum solution is calculated out quickly and accurately. Then, the simulation experiments are carried out and the detection error are analyzed quantitatively. The experiment results show that the improved algorithm can update the velocities and positions of particles in real-time. Compared with the original one, the improved algorithm can obtain the global optimum solution quickly and the accuracy of detection image has been improved more obviously.

Future work may focus on the following aspects. For further applications, lean percentage should be adjusted to change the swarm size, the the most effective particle swarm will be gained. Beyond, penalty function can also be added to transform the optimal problem into unconstrained, so that the variable range of the particle swarm can be restricted to constraint cluster.

\section{Acknowledgements}

This research is supported by National Natural Science Foundation of China $(11372199,51208318)$ and Natuab Science Poundation of Hebei Province (A2014210142).

\section{References}

[1] Y. Shufen and C. Jianzheng, Study on intage detection approach to wheel-rail contact points”, Electric Locomotives \& Mass Transit Vehicles, vol. no. 32, (2009).

[2] M. Jianjun, Y. Yông, Z. Yanlong and X. Ruxun, "Research on monitoring and virtual test for hunting motion of trains", Jounal of Railway Science and Engineering, vol. 5, no. 11, (2014).

[3] N. Aggarwal and W. C. Karl, "Line detection in images through regularized Hough transform", IEEE Transactions on Image Processing, vol. 15, no. 3, (2006), pp. 582-591.

[4] K. Hahn S. Jung and X. Lan, "A new algorithm for ellipse detection by curve segments", Pattern Recognition Letter, vol 29, no. 13, (2008), pp. 1836-1841.

[5] A. Bonci, T. Leo and Songhi, "A Bayesian approach to the Hough transform for line detection", IEEE Transactions on System s, Man, and Cybernetics, vol. 35, no. 6, (2005), pp. 945-955.

[6] V. Leemans and M. F. Destain, "Application of the Hough transform for seed row localization using machine vision", Bio-systems Engineering, vol. 94, no. 3, (2006), pp. 325-336.

[7] L. Xu, "A unified perspective and new results on RHT computing, mixture based learning, and multilearner based problem solving", Pattern Recognition, vol. 40, no. 8, (2007), pp. 2129-2153.

[8] E. Duquenoy and A. T. Ahmed, "Applying the Hough transform pseudo-linearity property to improve computing speeding”, Pattern Recognition Letters, vol. 27, no. 16, (2006), pp. 1893-1904.

9] Z. Yingtao, H. J. Hua, T. X. Long and L. J. Feng, "A hough transform algorithm based on reduced particle swarm optimization”, Journal of tianjin university, vol. 44, no. 2, (2011).

[10] Z. H. Dong and Z. Yong, "A kind of Rene wed simulated annealing algorithm", Computer technology and development, vol. 6, no. 19, (2009).

[11] L. Juan, D. Qingliang, Y. Hui and Z. Yiwen, "Parallel simulated annealing algorithm based on particle swarm optimization algorithm", Journal of frontiers of computer science and technology, vol. 8, no. 7, (2014).

[12] C. Galambos, J. Kittler and J. Matas, "Using gradient information to enhance the progressive probabilistic Hough transform", Proceedings of the International Conference on Pattern Recognition. Barcelona, Spain, (2000), pp. 560-563.

[13] J. Matas, C. Galambos and J. Kittler, "Progressive probabilistic Hough Transform", Proceedings of British Machine Vision Conference. Southampton, UK, (1998), pp. 256-265. 
[14] Z. Cheng and Y. Liu, "Efficient technique for ellipse detection using restricted randomized Hough Transform", Proceedings of International Conference on Information Technology. Las vegans, USA, (2004), pp. 714-718.

[15] J. Kennedy and R. Eberhar, "Particle swarm optimization", in: Proceedings of the 4th IEEE International Conference on Neural Networks, Piscataway: IEEE Service Center, (1995), pp. 1942 -1948.

[16] G. Qinghua, "Study on the derailment mechanism and running safety of trains", Southwest Jiao tong University, (2011).

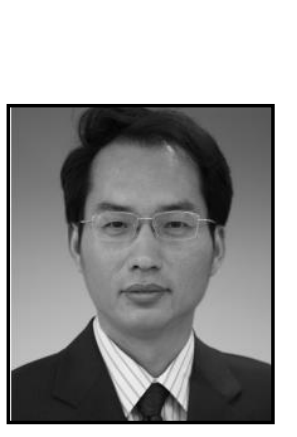

\section{Authors}

Zengqiang Ma, Born in Hebei province of China, 1975. Ph.D. degree was earned in major of vehicle engineering, Beijing Jiaotong University in 2011. Major Field of study is digital signal processing. $\mathrm{He}$ has published many papers in recent years and more than ten of them were included in the international Engineering Index (EI). Current and previous research interests include signal processing and network measurement \& control system.

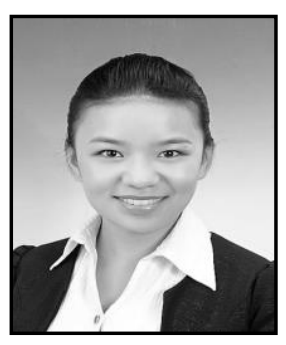

Xiaoyun Liu, She received her Bâchelor in Electrical Engineering (2014) and is a postgraduate in Shifiazhuang Tiedao University. Her current research interests include different aspects of Signal Processing.

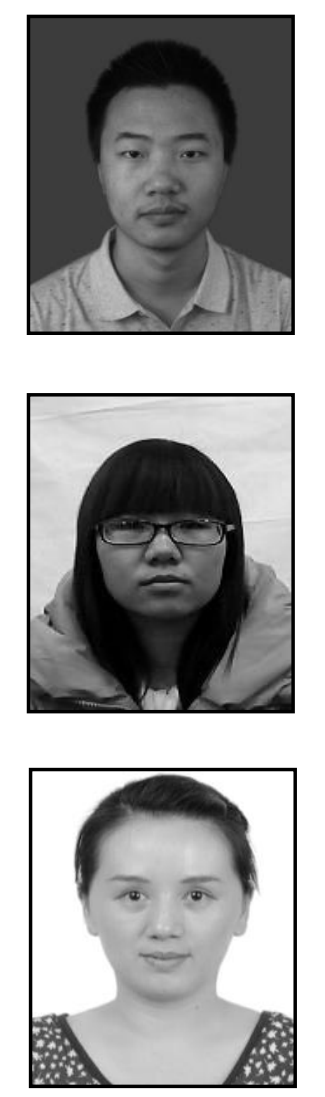

Zheng Liu, He received his Bachelor in Electrical Engineering (2013) and is postgradate in Shijiazhuang Tiedao University. His current research interests include different aspects of Signal Processing.

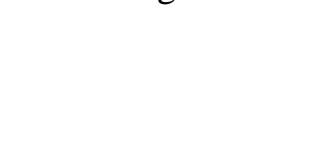

Sha Zhong, She received her Bachelor in Electrical Engineering (2013) and is a postgraduate in Shijiazhuang Tiedao University. Her carrent research interests include different aspects of Signal Processing.

Yusi Zhang, She received her Bachelor in management degree (2004) and is a teacher in Hebei Jiaotong Vocational and Technical College. Her current research interests include different aspects of Signal Processing. 
International Journal of Multimedia and Ubiquitous Engineering

Vol.11, No.11 (2016)

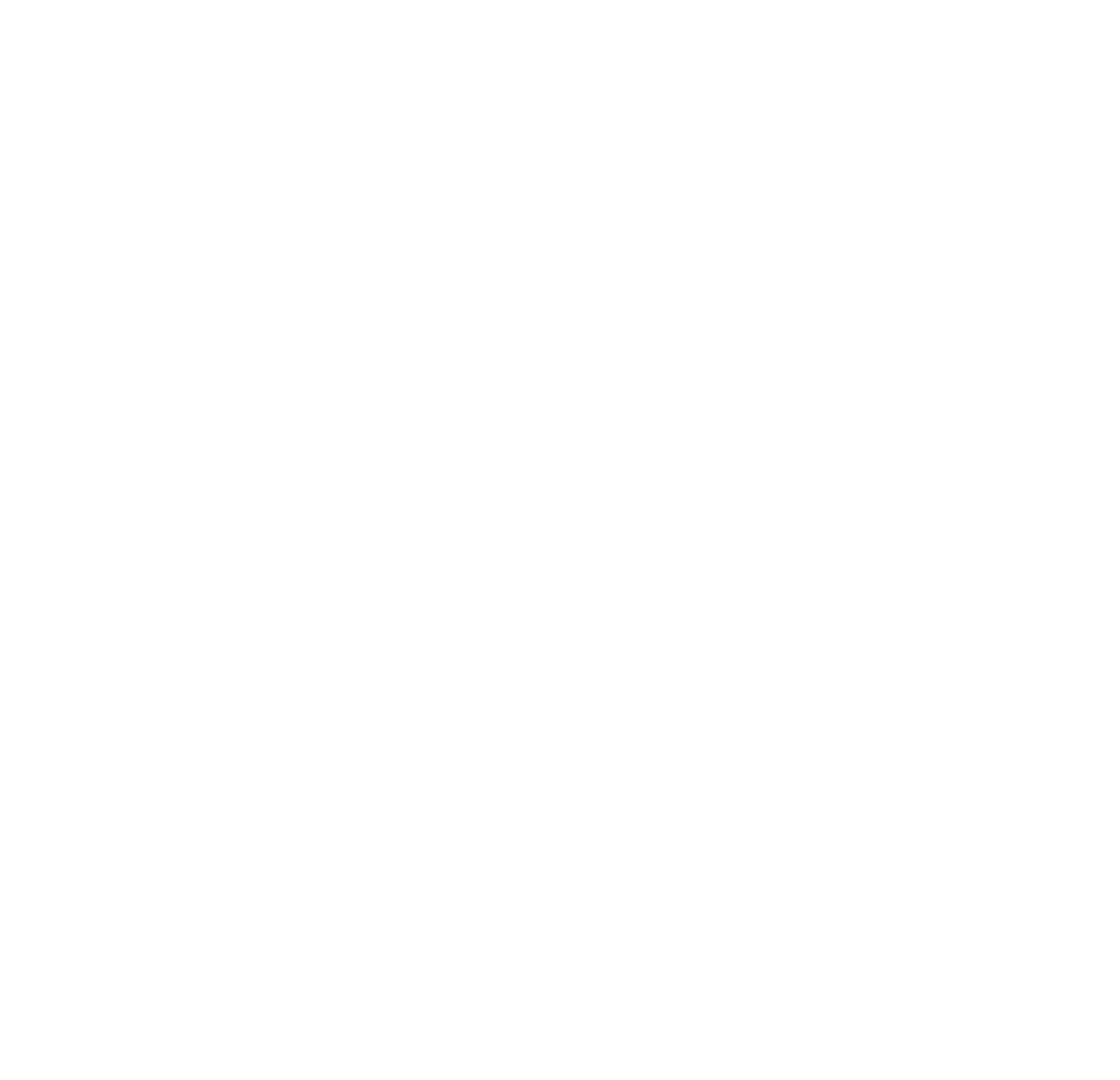

